\title{
THE REPRESENTATION-FINITE SYMMETRIC ALGEBRAS WITH LIFTABLE SIMPLE MODULES
}

\author{
G. MICHLER AND $\varnothing$. SOLBERG
}

\section{INTRODUCTION}

In [8] G. J. Janusz showed that a $p$-block algebra $\Lambda$ of a finite group $G$ of finite representation type over a splitting field $F$ with characteristic $p>0$ is uniserial if and only if every simple $\Lambda$-module $M$ can uniquely be lifted to a simple $\hat{\Lambda} \otimes_{R} S$ module, where $R$ is a complete discrete rank one valuation ring $R$ with maximal ideal $\pi R$, residue class field $F=R / \pi R$, and quotient field $S$ with characteristic zero, and where $\hat{\Lambda}$ is an $R G$-block such that $\Lambda \cong \hat{\Lambda} \otimes_{R} F$. If $\Lambda$ has at least two non-isomorphic simple modules, then using standard results of the theory of blocks with cyclic defect groups [5], p. 302, and J. A. Green's work [6] it can easily be seen that this liftability condition is equivalent to the requirements that $\operatorname{Ext}_{\Lambda}^{2}(M, M)=(0)=\operatorname{Ext}_{\Lambda}^{1}(M, M)$ for every simple $\Lambda$-module $M$.

These Ext-conditions also imply the unique liftability of the simple modules $M$ of an arbitrary finite-dimensional symmetric algebra $\Lambda$ over a commutative field $K$ in the sense of Auslander, Ding and the second author [1]. It is also kwown that $\operatorname{Ext}_{\Lambda}^{2}(M, M)=(0) \operatorname{implies}_{\operatorname{Ext}_{\Lambda}^{1}}(M, M)=(0)$. In order to generalize Janusz' theorem to the theory of finite dimensional symmetric algebras $\Lambda$ over an algebraically closed field $K$ it therefore suffices to classify all such connected algebras $\Lambda$ of finite representation type satisfying $\operatorname{Ext}_{\Lambda}^{2}(M, M)=(0)$ for all simple $\Lambda$-modules $M$. In this article we give such a classification.

In order to state it the following definitions and notations are needed. Let $J=$ $J(\Lambda)$ denote the radical of $\Lambda$ and let $\operatorname{soc}(X)$ denote the socle of a module $X$. If $P_{M}$ denotes the projective cover of the simple $\Lambda$-module $M$, then $H_{M}=P_{M} J / \operatorname{soc}\left(P_{M}\right)$ is called the heart of $P_{M}$. The $K$-algebra $\Lambda$ is said to have multiplicity $c(\Lambda)=1$ if $P_{M} J$ is a multiplicity-free $\Lambda$-module for every simple $\Lambda$-module $M$. Now we can state our main result.

Theorem 1.1. Let $\Lambda$ be a connected, representation-finite, symmetric algebra over the algebraically closed field $K$ with at least two non-isomorphic simple $\Lambda$-modules. Then the following conditions are equivalent:

(1) $\operatorname{Ext}_{\Lambda}^{2}(M, M)=(0)$ for every simple $\Lambda$-module $M$.

(2) Either $\Lambda$ is uniserial or $c(\Lambda)=1$ and the hearts $H_{M}$ of all indecomposable projective $\Lambda$-modules $P_{M}$ are indecomposable $\Lambda$-modules.

This result is proved in section 3 . The non uniserial algebras $\Lambda$ with multiplicity $c(\Lambda)=1$ satisfying condition (2) are classified by Theorem 4.1 in section 4 . This classification is based on the classification of all representation-finite connected basic symmetric algebras of Dynkin type $D_{n}$ by Bretscher, Läser and Riedtmann [4], and Roggon's description [11] of the quivers and relations of the basic symmetric algebras of Dynkin types $E_{6}, E_{7}$ and $E_{8}$.

In section 1 the relation between the module structure of the heart $H_{M}$ of the projective cover $P_{M}$ of the simple $\Lambda$-module $M$ and the condition $\operatorname{Ext}_{\Lambda}^{2}(M, M)=$ 
(0) is studied. These subsidiary results are the main new ingredients of the proof of Theorem 1.1.

Concerning our terminology and notation we refer to the books by Auslander, Reiten, Smalø [2] and Feit [5].

\section{Hearts of IndECOMPOSABLE PROJECTIVES}

This section is devoted to showing that if $\operatorname{Ext}_{\Lambda}^{2}(M, M)=(0)$ for a simple module $M$ over a weakly symmetric artin algebra $\Lambda$, then the heart $H_{M}$ of the projective cover $P_{M}$ of $M$ is indecomposable. This is a special case of a more general result for artin algebras stating that for an indecomposable projective module $P$ with simple socle, then if $\operatorname{Ext}_{\Lambda}^{2}(P / P J, \operatorname{soc}(P))=(0)$, the heart $P J / \operatorname{soc}(P)$ is zero or indecomposable. For weakly symmetric finite dimensional algebras over an algebraically closed field we prove a converse statement.

We first discuss the general case for artin algebras.

Proposition 2.1. Let $\Lambda$ be an artin algebra and suppose that $P$ is an indecomposable projective $\Lambda$-module with simple socle. If $\operatorname{Ext}_{\Lambda}^{2}(P / P J, \operatorname{soc}(P))=(0)$ and $P J / \operatorname{soc}(P)$ is nonzero, then the heart $P J / \operatorname{soc}(P)$ is indecomposable.

Proof. Assume that $P / P J=M$ and $\operatorname{soc}(P)=M^{\prime}$ for some simple $\Lambda$-modules $M$ and $M^{\prime}$. We have that $\operatorname{Ext}_{\Lambda}^{2}\left(M, M^{\prime}\right) \simeq \operatorname{Ext}_{\Lambda}^{1}\left(P J, M^{\prime}\right)$. Since $\operatorname{soc}(P J)=M^{\prime}$, we have that $I(P J)=I\left(M^{\prime}\right)$, where $I(X)$ denotes the injective hull of the $\Lambda$-module $X$. Moreover, we have the exact sequence

$$
0 \rightarrow\left(P J, M^{\prime}\right) \rightarrow\left(P J, I\left(M^{\prime}\right)\right) \rightarrow\left(P J, I\left(M^{\prime}\right) / M^{\prime}\right) \rightarrow \operatorname{Ext}_{\Lambda}^{2}\left(M, M^{\prime}\right) \rightarrow 0 .
$$

Suppose that $P J / M^{\prime}=X \oplus Y$, where both $X$ and $Y$ are nonzero. Then consider the following composition of maps

$$
\alpha: P J \rightarrow P J / M^{\prime}=X \oplus Y \rightarrow X \rightarrow I\left(M^{\prime}\right) / M^{\prime},
$$

where all the maps are the canonical projections or inclusions. We want to show that the homomorphism $\alpha$ does not factor through the homomorphism $\pi: I\left(M^{\prime}\right) \rightarrow$ $I\left(M^{\prime}\right) / M^{\prime}$ and therefore represents a nonzero element in $\operatorname{Ext}_{\Lambda}^{2}\left(M, M^{\prime}\right)$.

Suppose that there is a map $\beta: P J \rightarrow I\left(M^{\prime}\right)$ such that $\alpha=\pi \circ \beta$. Let $x$ be in $P J$ such that $x+M^{\prime}$ is in $X \backslash X J$. There exists an element $r_{x}$ in $J$ such that $x \cdot r_{x}$ is a nonzero element in $\operatorname{soc}(P J)=M^{\prime}$. Moreover, $\alpha(x)=x+M^{\prime}=\pi \circ \beta(x)$, so that $\beta(x)=x+m$ for some $m$ in $M^{\prime}$. Therefore $\beta\left(x r_{x}\right)=\beta(x) r_{x}=(x+m) r_{x}=x r_{x} \neq 0$. Hence, $\beta(s) \neq 0$ for any element $s$ in $\operatorname{soc}(P J)=M^{\prime}$.

Let $y$ be in $P J$ such that $y+M^{\prime}$ is in $Y \backslash Y J$. There exists an element $r_{y}$ in $J$ such that $y \cdot r_{y}$ is a nonzero element in $\operatorname{soc}(P J)=M^{\prime}$. Hence $\beta\left(y r_{y}\right) \neq 0$ and $\beta(y) \neq 0$. If $\beta(y)$ is in $M^{\prime}$, then $0=\beta(y) r_{y}=\beta\left(y r_{y}\right)$. This is impossible, so that $\pi \circ \beta(y) \neq 0$. Since $\alpha(y)=\pi \circ \beta(y)=0$, we have a contradiction. Therefore the map $\alpha$ defined as above does not factor through the homomorphism $\pi: I\left(M^{\prime}\right) \rightarrow I\left(M^{\prime}\right) / M^{\prime}$. From the above exact sequence follows that $\operatorname{Ext}_{\Lambda}^{2}\left(M, M^{\prime}\right) \neq(0)$. This completes the proof.

For weakly symmetric artin algebras all indecomposable projectives have simple socles. In this case we have the following corollary which we apply in section 3 .

Corollary 2.2. Let $\Lambda$ be a weakly symmetric artin algebra and $M$ a nonprojective simple $\Lambda$-module. If $\operatorname{Ext}_{\Lambda}^{2}(M, M)=(0)$, the heart $H_{M}$ is indecomposable. In particular, the length of $P_{M}$ is at least 3 . 
Proof. If $H_{M}$ were zero, then we would have an exact sequence of the form $0 \rightarrow$ $M \rightarrow P_{M} \rightarrow M \rightarrow 0$. Therefore $\operatorname{Ext}_{\Lambda}^{1}(M, M) \cong \operatorname{Ext}_{\Lambda}^{2}(M, M)=0$, a contradiction to $M$ not being projective. Thus $H_{M}$ is nonzero. The claim follows from the previous lemma, and the length of $P_{M}$ is at least 3 .

In view of the above corollary it is natural to ask if $\operatorname{Ext}_{\Lambda}^{1}(M, M)=(0)$ and $H_{M}$ being indecomposable for a simple module $M$ over a weakly symmetric artin algebra $\Lambda$ implies that $\operatorname{Ext}_{\Lambda}^{2}(M, M)=(0)$. This is not the case as the following example shows.

Let $Q$ be the following quiver

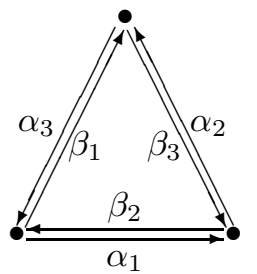

and $K$ a field. Let $I$ be the ideal in the path algebra $K Q$ generated by the elements $\left\{\alpha_{i-1} \beta_{i}-\beta_{i+1} \alpha_{i}, \alpha_{i+2} \alpha_{i+1} \alpha_{i}, \beta_{i+2} \beta_{i+1} \beta_{i}\right\}_{i=1}^{3}$, where the indices are calculated modulo 3 . Then it is easy to see that the algebra $\Lambda=K Q / I$ is weakly symmetric, all the hearts of the indecomposable projective $\Lambda$-modules are indecomposable and

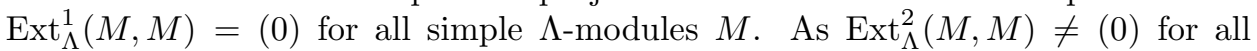
simple $\Lambda$-modules $M$, we see that the converse of Corollary 2.2 is by no means true.

A partial converse of Corollary 2.2 is the following.

Proposition 2.3. Let $\Lambda$ be a weakly symmetric finite dimensional algebra over a field $K$. Let $M$ be a simple $\Lambda$-module such that $\operatorname{End}_{\Lambda}\left(H_{M}\right)=K$ and $\operatorname{Ext}_{\Lambda}^{1}(M, M)=$ (0). Then $\operatorname{Ext}_{\Lambda}^{2}(M, M)=(0)$.

Proof. Let $M$ be a simple $\Lambda$-module such that $\operatorname{End}_{\Lambda}\left(H_{M}\right)=K$ and $\operatorname{Ext}_{\Lambda}^{1}(M, M)=$ (0). Since $\Lambda$ is selfinjective, we have that

$$
\operatorname{Ext}_{\Lambda}^{2}(M, M) \simeq \operatorname{Ext}_{\Lambda}^{1}\left(P_{M} J, M\right) \simeq \underline{\operatorname{Hom}}_{\Lambda}\left(P_{M} J, P_{M} / M\right) .
$$

We want to show that $\underline{\operatorname{Hom}}_{\Lambda}\left(P_{M} J, P_{M} / M\right)=(0)$. Let $\alpha: P_{M} J \rightarrow P_{M} / M$ be a $\Lambda$-homomorphism. Since $\operatorname{Ext}_{\Lambda}^{1}(M, M)=(0)$, the simple module $M$ is neither a composition factor of $P_{M} J / P_{M} J^{2}$ nor of $\operatorname{soc}\left(P_{M} / M\right)$. As $\alpha\left(P_{M} J\right) / \alpha\left(P_{M} J\right) J$ is a factor module of $P_{M} J / P_{M} J^{2}$, it follows that $\alpha\left(P_{M} J\right) \subset P_{M} J / M$. Since $\alpha\left(\operatorname{soc}\left(P_{M} J\right)\right) \subset$ $\operatorname{soc}\left(P_{M}\right) / M$, we have that $\operatorname{soc}\left(P_{M} J\right)=M$ is contained in ker $\alpha$. Therefore every homomorphism $\alpha: P_{M} J \rightarrow P_{M} / M$ induces an endomorphism $\bar{\alpha}$ of $H_{M}$. It follows that $\varphi: \operatorname{End}_{\Lambda}\left(H_{M}\right) \rightarrow \operatorname{Hom}_{\Lambda}\left(P_{M} J, P_{M} / M\right)$ given by $\varphi(\beta)=\nu \circ \beta \circ \pi$ for $\beta$ in $\operatorname{End}_{\Lambda}\left(H_{M}\right)$ is an isomorphism, where $\pi: P_{M} J \rightarrow H_{M}$ and $\nu: H_{M} \rightarrow P_{M} / M$ are the natural homomorphisms. Since $\nu \circ \pi: P_{M} J \rightarrow P_{M} / M$ factors through the natural projection $P_{M} \rightarrow P_{M} / M$ and $\operatorname{End}_{\Lambda}\left(H_{M}\right)=K$, it follows that any homomorphism $\alpha: P_{M} J \rightarrow P_{M} / M$ factors through $P_{M}$ and therefore $\operatorname{Ext}_{\Lambda}^{2}(M, M)=(0)$.

A situation where the heart $H_{M}$ of $P_{M}$ for a simple module $M$ satisfies the hypothesis of the previous result is the following. We apply this in section 3 .

Corollary 2.4. Let $\Lambda$ be a weakly symmetric finite dimensional algebra over an algebraically closed field $K$. Let $M$ be a simple $\Lambda$-module such that $P_{M} J$ is a multiplicity free $\Lambda$-module and the heart $H_{M}$ of $P_{M}$ is indecomposable. Then $\operatorname{Ext}_{\Lambda}^{2}(M, M)=(0)$. 
Proof. As $H_{M}$ is indecomposable $E=\operatorname{End}_{\Lambda}\left(H_{M}\right)$ is a finite dimensional local $K$ algebra. Hence $E / J(E) \simeq K$. Suppose there is a nonzero element $\alpha$ in $J(E)$. Then $\alpha$ is nilpotent. Hence both $\operatorname{ker} \alpha$ and $\alpha\left(H_{M}\right)$ are nonzero proper submodules of $H_{M}$ containing together all composition factors of $H_{M}$ because $\alpha\left(H_{M}\right) \simeq H / \operatorname{ker} \alpha$. As $H_{M}$ is multiplicity free $\alpha\left(H_{M}\right) \cap \operatorname{ker} \alpha=(0)$. Hence $H_{M}=\alpha\left(H_{M}\right) \oplus \operatorname{ker} \alpha$, a contradiction to $H_{M}$ being indecomposable. Thus $\operatorname{End}_{\Lambda}\left(H_{M}\right)=K$ and the claim follows from the previous result.

The condition of $\operatorname{Ext}_{\Lambda}^{2}(M, M)$ being zero also has come up in work on liftability of modules in [1]. We recall the notion of a lifting of a module from [1].

Let $\varphi: \Gamma \rightarrow \Lambda$ be a homomorphism of rings and let $M$ be a finitely generated $\Gamma$-module. Then finitely generated $\Gamma$-module $L$ is called a lifting of $M$ to $\Gamma$ if $M \simeq L \otimes_{\Gamma} \Lambda$ and $\operatorname{Tor}_{i}^{\Gamma}(L, \Lambda)=(0)$ for all $i \geq 1$. The $\Lambda$-module $M$ is said to be liftable to $\Gamma$ when such a $\Gamma$-module $L$ exists.

It is shown in Proposition 1.7 and Proposition 2.6 of [1] that if $\varphi: \Gamma \rightarrow \Lambda$ with $\Gamma$ and $\Lambda$ suitably given, then for a $\Lambda$-module $X$ the condition $\operatorname{Ext}_{\Lambda}^{2}(X, X)=(0)$ implies that $X$ is liftable to $\Gamma$. Furthermore, if a $\Lambda$-module $X$ is liftable to $\Gamma$ with $\operatorname{Ext}_{\Lambda}^{1}(X, X)=(0)$, then the lifting of $X$ to $\Gamma$ is unique.

In the following let $\Lambda$ be an artin algebra. Next we show that if $\operatorname{Ext}_{\Lambda}^{2}(M, M)=$ (0) for a simple $\Lambda$-module $M$, then $\operatorname{Ext}_{\Lambda}^{1}(M, M)=(0)$. Hence the sufficient condition $\operatorname{Ext}_{\Lambda}^{2}(M, M)=(0)$ for liftability of a simple $\Lambda$-module $M$ implies uniquely liftability.

Let $M$ be a simple $\Lambda$-module. Denote by $\tau_{X}(Y)$ the trace of $X$ in $Y$ for two $\Lambda$-modules $X$ and $Y$, that is,

$$
\tau_{X}(Y)=\sum_{\varphi \in \operatorname{Hom}_{\Lambda}(X, Y)} \varphi(X) .
$$

Let $\left\{M_{1}, M_{2}, \ldots, M_{n-1}, M_{n}=M\right\}$ be a complete set of nonisomorphic simple $\Lambda$ modules and let $Q=\oplus_{i=1}^{n-1} P_{M_{i}}$. For a $\Lambda$-module $A$ let $A(M)=A / \tau_{Q}(A)$. Then it is clear that the only simple occurring as a composition factor in $A(M)$ is $M$ and that $A(M)=(0)$ if and only if $M$ does not occur in the top, $A / A J$, of $A$. Moreover, $Q \simeq e \Lambda$ for some idempotent $e$ and $\tau_{Q}(A)=A(\Lambda e \Lambda)$ for a $\Lambda$-module $A$. Therefore we have that $A(M)=A \otimes_{\Lambda} \Lambda / \Lambda e \Lambda$ for a $\Lambda$-module $A$ and in particular it gives rise to a right exact functor.

The corollary to the following result is known. For the convenience of the reader we include a proof.

Proposition 2.5. Suppose $M$ is a simple $\Lambda$-module and $X$ is a $\Lambda$-module such that $X(M)$ is a nonprojective $\Lambda(M)$-module. Then $\operatorname{Ext}_{\Lambda}^{1}(X, M) \neq(0)$.

Proof. Let $M$ be a simple $\Lambda$-module and assume that $X(M)$ is a nonprojective $\Lambda(M)$-module. Now $\operatorname{Ext}_{\Lambda}^{1}(X, M) \neq(0)$ if and only if $\operatorname{Hom}_{\Lambda}(\Omega(X) / \Omega(X) J, M) \neq$ $(0)$, where $\Omega$ denotes the Heller operator.

We have the following exact commutative diagram.

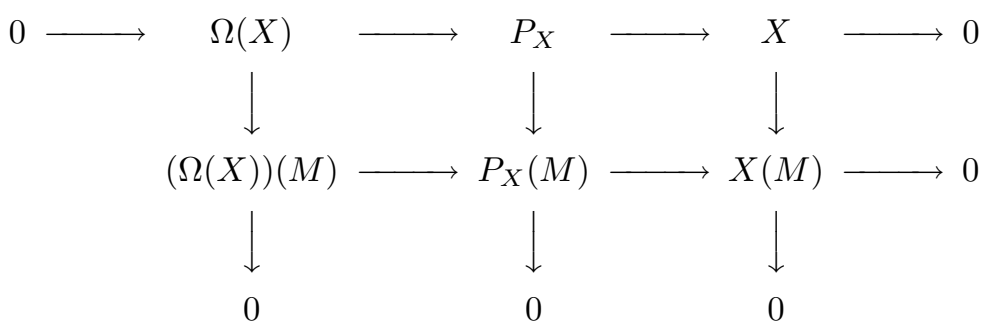

The map $\varphi: P_{X}(M) \rightarrow X(M)$ is a projective cover. Since $X(M)$ is a nonprojective $\Lambda(M)$-module, $\operatorname{ker} \varphi$ is a nonzero $\Lambda(M)$-module. Therefore $(\Omega(X))(M)$ must be 
nonzero. Hence $M$ occurs in the top of $\Omega(X)$. The claim follows from this by the above observation.

Corollary 2.6. Let $M$ be a simple $\Lambda$-module. Then $\operatorname{Ext}_{\Lambda}^{2}(M, M)=(0)$ implies that $\operatorname{Ext}_{\Lambda}^{1}(M, M)=(0)$.

Proof. Assume that $\operatorname{Ext}_{\Lambda}^{1}(M, M) \neq(0)$. Then we know that $M$ occurs as a composition factor in the top $\Omega(M)=P_{M} J$. It is easy to see that $0 \rightarrow\left(P_{M} J\right)(M) \rightarrow$ $P_{M}(M) \rightarrow M \rightarrow 0$ is exact. Therefore $\left(P_{M} J\right)(M)$ is a nonprojective $\Lambda$-module and $\operatorname{Ext}_{\Lambda}^{1}\left(P_{M} J, M\right) \simeq \operatorname{Ext}_{\Lambda}^{2}(M, M) \neq(0)$. This completes the proof of the corollary.

\section{Proof of the MAIN RESUlT}

In this section we assume that all algebras $\Lambda$ are finite dimensional connected symmetric algebras over an algebraically closed field $K$.

Let $\left\{e_{i}\right\}_{i=1}^{n}$ be a complete set of primitive orthogonal idempotents for $\Lambda$. Recall that $\Lambda$ is called regular if for each pair of $i$ and $j$ there exists $x$ and $y$ in $e_{i} \Lambda e_{j}$ such that $e_{i} \Lambda e_{j}=e_{i} \Lambda e_{i} x$ and $e_{i} \Lambda e_{j}=y e_{j} \Lambda e_{j}$. Moreover, the multiplicity of $\Lambda$ is defined by $c(\Lambda)=\max \left\{\operatorname{dim}_{K} e_{i} \Lambda e_{i}\right\}_{i=1}^{n}-1$.

Proof of Theorem 1.1. Let $\Lambda$ be a connected, representation-finite, symmetric $K$ algebra with at least two non-isomorphic simple modules such that $\operatorname{Ext}_{\Lambda}^{2}(M, M)=$ (0) for every simple $\Lambda$-module $M$. Since all these properties of $\Lambda$ are preserved under Morita equivalence we may assume that $\Lambda$ is a basic $K$-algebra.

If $\Lambda$ is not regular, then by [13, Satz 1.8] the multiplicity of $\Lambda$ is at least 2 . Hence by [13, Satz 1] $\Lambda$ is isomorphic to one of the algebras which are denoted in [13] by $B(T, S, \epsilon)$, where $T$ is a Brauer tree, $S$ an extremal point on $T$ and the scalar $\epsilon$ is 0 or 1 . Since $\Lambda$ has at least two simple modules, it follows from the description of the quivers and relations of the algebras $B(T, S, \epsilon)$ that there is a simple $\Lambda$-module $M$ such that $M$ occurs in the top of $P_{M} J$. From Corollary 2.6 follows that $\operatorname{Ext}_{\Lambda}^{2}(M, M) \neq(0)$, a contradiction.

Hence we may assume that $\Lambda$ is regular. If the multiplicity of $\Lambda$ is at least 2 , then by [13, Satz 1] and [12, Corollary a] we have that $\Lambda$ is biserial. By Corollary 2.2 the hearts $H_{M}$ of all indecomposable projective $\Lambda$-modules $P_{M}$ are indecomposable. Therefore $\Lambda$ is uniserial.

Thus we may assume that $\Lambda$ is regular with multiplicity $c(\Lambda)=1$. Therefore assertion (2) of Theorem 1.1 is a consequence of assertion (1).

The proof of the converse is split into two parts. At first we assume that $\Lambda$ is uniserial. Since $\Lambda$ has at least two non-isomorphic simple modules, it follows from Corollary 2.12 of [2], p. 117, that all simple $\Lambda$-modules are contained in one orbit of the square $\Omega^{2}$ of the Heller operator $\Omega$. In particular, $\operatorname{Ext}_{\Lambda}^{2}(M, M)=$ $\operatorname{Hom}_{\Lambda}\left(\Omega^{2} M, M\right)=(0)$ for every simple $\Lambda$-module.

Now we assume that $\Lambda$ has multiplicity $c(\Lambda)=1$ and all hearts $H_{M}$ of the indecomposable projective $\Lambda$-modules are indecomposable. Then $P_{M} J$ is a multiplicityfree $\Lambda$-module for each simple $\Lambda$-module $M$ by Satz 1.1 of [9] and Theorem 2 of [10] because $\Lambda$ is of finite representation type. Hence $\operatorname{Ext}_{\Lambda}^{2}(M, M)=(0)$ for all simple $\Lambda$-modules $M$ by Corollary 2.4. This completes the proof. 


\section{The Classification of the NON-Uniserial BASIC ALGEBRAS}

This section is devoted to classifying all representation-finite non-uniserial connected basic symmetric $K$-algebras $\Lambda$ with $\operatorname{Ext}_{\Lambda}^{2}(M, M)=(0)$ for all simple $\Lambda$ modules $M$ where $K$ as the classification is based on the classification of all representation-finite connected basic symmetric algebras by Bretscher, Läser and Riedtmann [4] and Roggon's [11] description of the quivers and relations of the connected basic symmetric algebras of Dynkin types $E_{6}, E_{7}$ and $E_{8}$.

First we recall how to construct the quivers and relations from the data given for the cases $E_{6}, E_{7}$ and $E_{8}$ listed below.

The ordinary quiver $\Gamma$ of an representation-finite symmetric algebra of Dynkin type $E_{n}$ has $n$ vertices. The sequence of numbers in the last column in the table, for example 142434454651536162 , describes the arrows in $\Gamma$, that is, there are arrows $1 \rightarrow 4,2 \rightarrow 4,3 \rightarrow 4$ and so on. We let $Q$ be the quiver obtained from $\Gamma$ by only including the arrows $i \rightarrow j$ where $i<j$. By $\mathbb{Z} Q$ we denote the infinite quiver given by the Cartesian produkt $Q \times \mathbb{Z}$. Let $\Delta$ be the infinite quiver obtained from $\mathbb{Z} Q$ by adding arrows $(i, n) \rightarrow(j, n+1)$ if there is an arrow $j \rightarrow i$ in $\Gamma$ with $i>j$. Let $\nu: \Delta \rightarrow \Delta$ be the automorphism given by $\nu((i, n))=(i, n+1)$ and $\nu(\alpha:(i, n) \rightarrow(j, m))=\alpha:(i, n+1) \rightarrow(j, m+1)$, when $m=n$ or $m=n+1$.

Let $I$ be the ideal in $K \Delta$ generated by all full commutativity relations and all paths $p: x \leadsto y$, unless there is an extension $q$ such that $q p: x \leadsto y \leadsto \nu(x)$. Then the algebra given by the sequence of numbers in the last column in the table below for $E_{n}$ is $(K \Delta / I) / \nu^{\mathbb{Z}}$. The lower case letter $s$ next to the number in the first column in the list indicates that the algebra $\Lambda$ is selfdual, i.e. $\Lambda \cong \Lambda^{o p}$.

Now we can state the classification. If the algebra $\Lambda$ is not self-dual, only one of the algebras $\Lambda$ and $\Lambda^{o p}$ is listed. We keep Roggon's numbering [11] of the $K$ algebras of Dynkin types $E_{6}, E_{7}$ and $E_{8}$.

Theorem 4.1. Let $\Lambda$ be a non-uniserial symmetric, connected, representationfinite, basic $K$-algebra with mulitplicity $c(\Lambda)=1$ such that $\operatorname{Ext}_{\Lambda}^{2}(M, M)=(0)$ for all simple $\Lambda$-modules. If $\Lambda$ is not semi-simple, then $\Lambda$ has at least 2 non-isomorphic simple $\Lambda$-modules, and it is either of Dynkin type $D_{n}, E_{6}, E_{7}$ or $E_{8}$.

Furthermore the following statements hold:

(1) If $\Lambda$ is of Dynkin type $D_{n}$, then its quiver has $n$ vertices, and it is either

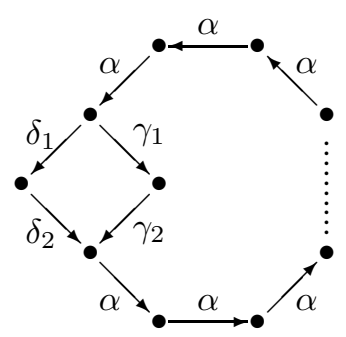

with $n$ relations generated by the elements $\gamma_{2} \gamma_{1}-\delta_{2} \delta_{1}, \gamma_{1} \alpha^{n-3} \delta_{2}, \delta_{1} \alpha^{n-3} \gamma_{2}$ and $\alpha^{n-2-i} \gamma_{2} \gamma_{1} \alpha^{i}$ for $i=1, \ldots, n-3$, or it is 


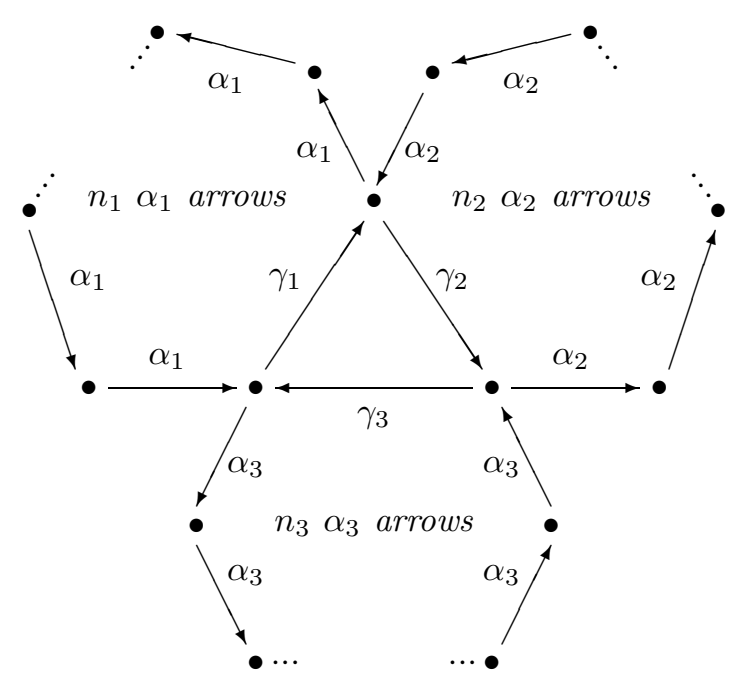

where $n_{1}+n_{2}+n_{3}=n \geq 5, n_{i} \geq 1$, and where the relations are generated by the elements $\gamma_{3} \gamma_{2}-\alpha_{1}^{n_{1}}, \gamma_{1} \gamma_{3}-\alpha_{2}^{n_{2}}, \gamma_{2} \gamma_{1}-\alpha_{3}^{n_{3}}, \alpha_{2} \alpha_{3}, \alpha_{1} \alpha_{2}, \alpha_{3} \alpha_{1}, \alpha_{j}^{n_{j}-i} \gamma_{j} \alpha_{j}^{i+1}$ for $i=0,1, \ldots, n_{j}-1$ and $j=1,2,3$.

(2) There are 9 non-isomorphic such algebras $\Lambda$ of Dynkin type $E_{6}$. Their quivers and relations are given in the following table.

\begin{tabular}{|c|c|l|}
\hline Number & Dimension & Quiver and relations \\
\hline 10 & 30 & 142434454651536162 \\
\hline 13 & 30 & 121423253631455162 \\
\hline 14 & 30 & 121324344546415362 \\
\hline 17 & 32 & 1215232436415661 \\
\hline 18 & 34 & 1214232531455661 \\
\hline 19 & 34 & 1215233436415661 \\
\hline 20 & 34 & 12132534465661 \\
\hline 21 & 36 & 1223253436415661 \\
\hline 22 & 38 & 12233436455161 \\
\hline
\end{tabular}

(3) There are 41 non-isomorphic such algebras $\Lambda$ of Dynkin type $E_{7}$. Their quivers and relations are given in the following table.

\begin{tabular}{|c|c|c|c|}
\hline Number & Dimension & Quiver and relations \\
\hline $36 \mathrm{~s}$ & 36 & 1214162325314547516771 \\
\hline 39 & 36 & 1214232531454657516172 \\
\hline $49 \mathrm{~s}$ & 36 & 1214232536314551676273 \\
\hline 53 & 38 & 12152326344156676175 \\
\hline 57 & 38 & 12152324364741566172 \\
\hline 58 & 38 & 12152326344156576171 \\
\hline 59 & 38 & 12141623273545516771 \\
\hline 61 & 40 & 12152334364156576171 \\
\hline 63 & 40 & 12152326344741566172 \\
\hline $67 \mathrm{~s}$ & 40 & 12152324364156676172 \\
\hline $68 \mathrm{~s}$ & 40 & 12152334364741566173 \\
\hline 70 & 40 & 121623253441576771 \\
\hline 72 & 42 & 12162324353745516771 \\
\hline \multicolumn{4}{|c|}{} \\
\hline
\end{tabular}




\begin{tabular}{|c|c|l|l|}
\hline \multicolumn{3}{|c|}{ continued from previous page } \\
\hline $73 \mathrm{~s}$ & 42 & 121623253447516771 \\
\hline 75 & 42 & 121523263441566771 \\
\hline $77 \mathrm{~s}$ & 42 & 12232534364156676173 \\
\hline $78 \mathrm{~s}$ & 44 & 12152326343741566771 \\
\hline 79 & 44 & 121623343547516771 \\
\hline 81 & 44 & 121623343745516771 \\
\hline $82 \mathrm{~s}$ & 44 & 1223263445516771 \\
\hline 83 & 46 & 122325344656576171 \\
\hline 84 & 46 & 121623344547516771 \\
\hline 85 & 48 & 122325343641566771 \\
\hline $86 \mathrm{~s}$ & 50 & 1223343745566171 \\
\hline $87 \mathrm{~s}$ & 50 & 122334364547516771 \\
\hline $88 \mathrm{~s}$ & 52 & 1223344547566171 \\
\hline
\end{tabular}

(4) There are 139 non-isomorphic such algebras $\Lambda$ of Dynkin type $E_{8}$. Their quivers and relations are given in the following table.

\begin{tabular}{|c|c|c|}
\hline Number & Dimension & Quiver and relations \\
\hline 142 & 42 & 12142325373145465851617284 \\
\hline 143 & $\overline{42}$ & 12141623253145475851677182 \\
\hline 149 & 42 & 12142325373145465161787283 \\
\hline $150 \mathrm{~s}$ & 42 & 12142325273831454651617882 \\
\hline $153 \mathrm{~s}$ & 42 & 12142325363145585167627384 \\
\hline 158 & 44 & 121523263441565761787185 \\
\hline 159 & 44 & 121523263441566761787286 \\
\hline 164 & 44 & 121517232634415658617881 \\
\hline 167 & $\overline{44}$ & 121523263441565768617182 \\
\hline 176 & 44 & 121523243641565768617185 \\
\hline $185 \mathrm{~s}$ & 44 & 121523263437415661787283 \\
\hline 190 & 44 & 121523243648415657617182 \\
\hline 193 & 44 & 121523243647415661787284 \\
\hline 194 & 44 & 121423354546475168617884 \\
\hline 200 & 44 & 121416233545475851677183 \\
\hline $205 \mathrm{~s}$ & 44 & 12152334415667617885 \\
\hline 208 & 44 & 121423354546516761787486 \\
\hline $209 \mathrm{~s}$ & 44 & $12141623273545485167 \quad 7181$ \\
\hline 210 & 46 & 121523343641565761787185 \\
\hline 212 & 46 & 121523343641565768617185 \\
\hline 221 & 46 & 121523343647415661787384 \\
\hline 227 & 46 & 121523343648415657617183 \\
\hline 228 & 46 & $12151723 \quad 3436415658617881$ \\
\hline 230 & 46 & 1215232734384156677182 \\
\hline 234 & 46 & 1215232436415657617881 \\
\hline $241 \mathrm{~s}$ & 46 & 1216232534384157677182 \\
\hline 244 & 48 & 1216232734455167687181 \\
\hline $245 \mathrm{~s}$ & 48 & 12162325344158677881 \\
\hline 248 & 48 & 1215232734415667787185 \\
\hline 249 & 48 & 1215233446565761787185 \\
\hline 250 & 48 & 1215232634415667687182 \\
\hline \multicolumn{3}{|c|}{ continued on next page } \\
\hline
\end{tabular}




\begin{tabular}{|c|c|c|}
\hline \multicolumn{3}{|c|}{ continued from previous page } \\
\hline 254 & 48 & 121523343641565768617182 \\
\hline 258 & 48 & 1215233437415658677181 \\
\hline 259 & 48 & 121623243537455167687181 \\
\hline 260 & 48 & 1215232634415667687185 \\
\hline 261 & $\overline{48}$ & 121623243537455851677183 \\
\hline 264 & 48 & 1216233435474851677184 \\
\hline 265 & 48 & $\begin{array}{llllllllllll}12 & 14 & 23 & 25 & 36 & 45 & 47 & 56 & 61 & 78 & 71 & 84\end{array}$ \\
\hline 268 & 48 & 1214162335454751677881 \\
\hline 272 & 48 & 1216232534475167687181 \\
\hline $275 \mathrm{~s}$ & 50 & 1215232634415667787182 \\
\hline 277 & 50 & 1216232435384551677881 \\
\hline 278 & 50 & 1216233435475851677183 \\
\hline 279 & 50 & 1216233435475167687181 \\
\hline 282 & 50 & 1215232436484156677182 \\
\hline 285 & 50 & 12172326344551687881 \\
\hline $286 \mathrm{~s}$ & 50 & 1215232634484156677182 \\
\hline 287 & 50 & 121523263437415667687182 \\
\hline $288 \mathrm{~s}$ & 50 & 1216233437455167787183 \\
\hline 289 & 50 & 1217233435465668617184 \\
\hline 293 & 52 & 12162334384551677881 \\
\hline 295 & 52 & 1223263437455167787183 \\
\hline 296 & 52 & 1215232436415657687881 \\
\hline 298 & 52 & 12162327344551677881 \\
\hline 301 & 52 & 1217232534465658617881 \\
\hline 303 & 52 & 12162334354851677881 \\
\hline 305 & 52 & 1216233445475167687181 \\
\hline 306 & 52 & 1216232734455851677182 \\
\hline 307 & 54 & 1215232734384156677881 \\
\hline 309 & 54 & 1215233436475658677181 \\
\hline 312 & 54 & 1223253436415667687183 \\
\hline $315 \mathrm{~s}$ & 54 & 12172326344558617881 \\
\hline 316 & 56 & 1216232734454851677881 \\
\hline 318 & 56 & 12152334465658677181 \\
\hline 319 & 56 & 1214232536454856677181 \\
\hline $320 \mathrm{~s}$ & 56 & 12232634384551677881 \\
\hline 321 & 56 & $12 \quad 172334364558617881$ \\
\hline $323 \mathrm{~s}$ & 56 & 122327344556617881 \\
\hline 324 & 58 & 12232634455767687181 \\
\hline 325 & 58 & 12152324364156677881 \\
\hline 327 & 60 & $12232734454856 \quad 617881$ \\
\hline 328 & 60 & $121423253145 \quad 56 \quad 67 \quad 7881$ \\
\hline $329 \mathrm{~s}$ & 60 & $122326343745485167 \quad 7881$ \\
\hline 330 & 62 & 12232534364156677881 \\
\hline 331 & 64 & 12233437455658617881 \\
\hline $332 \mathrm{~s}$ & 64 & 122334384556677181 \\
\hline $333 \mathrm{~s}$ & 66 & 12233445475658617881 \\
\hline $334 \mathrm{~s}$ & 68 & 122334455658677181 \\
\hline
\end{tabular}


Proof. Let $\Lambda$ be a representation-finite connected basic symmetric $K$-algebra with multiplicity $c(\Lambda)=1$, where $K$ is an algebraically closed field. As $\Lambda$ is not semisimple it has at least two non-isomorphic simple modules. If $\Lambda$ were non-regular, then by [13, Satz 1.8] its multiplicity would be 3 , a contradiction to $c(\Lambda)=1$. Hence $\Lambda$ is regular. By $[7, \S 4.1] \Lambda$ is a trivial extension of a tilted algebra of Dynkin class $A_{n}, D_{n}, E_{6}, E_{7}$ or $E_{8}$. As $\Lambda$ is not uniserial, it is not of Dynkin class $A_{n}$. All the trivial extensions of the tilted algebras are standard [11, Proposition 2.3]. Therefore it remains to classify all standard representation-finite connected basic symmetric $K$-algebras of Dynkin class $D_{n}, E_{6}, E_{7}$ and $E_{8}$. Each case is now dealt with separately.

(1) Suppose that $\Lambda$ is of Dynkin class $D_{n}$. All standard representation-finite connected basic symmetric $K$-algebras of Dynkin class $D_{n}$ are classified in [4]. They are either two- or three-cornered algebras.

In [4] the two-cornered algebras of Dynkin class $D_{n}$ are given in the following way. An arrowed Brauer-quiver $Q$ is a finite connected quiver satisfying the conditions:

(a) each arrow of $Q$ belongs to a simple oriented cycle,

(b) each vertex belongs exactly to two cycles, and

(c) two cycles meet in at most one vertex. The arrows belonging to one cycle are all labeled by $\alpha$ or $\beta$ in such a way that two $\alpha$-cycles (or $\beta$-cycles) never meet.

Let $P$ be an arrowed Brauer-quiver with $n-2$ vertices and a distinguished arrow $\alpha: x \rightarrow y$. Let $a_{z}\left(b_{z}\right)$ be the length of the $\alpha$-cycle $A_{z}\left(\beta\right.$-cycle $\left.B_{z}\right)$ of $P$ passing through the vertex $z$. Then denote by $Q(P)$ the quiver obtained from $P$ replacing the distinguished arrow $\alpha: x \rightarrow y$ by

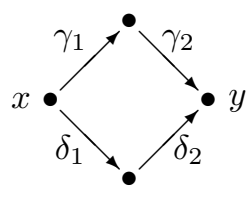

Let $I(P)$ be the ideal in $K Q(P)$ generated by the elements

(a) $\alpha \beta, \beta \alpha, \gamma_{1} \beta, \beta \gamma_{2}, \delta_{1} \beta, \beta \delta_{2}, \delta_{1} \alpha^{a_{x}-1} \gamma_{2}, \gamma_{1} \alpha^{a_{x}-1} \delta_{2}, \gamma_{2} \gamma_{1}-\delta_{2} \delta_{1}$

(b) $\alpha^{a_{z}}-\beta^{b_{z}}$ for each vertex $z$ of $P$ lying outside $A_{x}$.

(c) $\alpha^{a_{z}^{+}} \gamma_{2} \gamma_{1} \alpha^{a_{z}^{-}}-\beta^{b_{z}}$ for each vertex $z$ of $A_{x}$, where $a_{z}^{-}\left(a_{z}^{+}\right)$denote the length of the $\alpha$-path of $Q(P)$ from $z$ to $x$ ( $y$ to $z$ ).

Then the algebra $\Lambda=K Q(P) / I(P)$ is a two-cornered algebra and by [4] all twocornered algebras are given in this way.

Let $\Lambda$ be a two-cornered algebra. If there is an $\alpha$-cycle meeting a $\beta$-cycle both of length at least 2 , then the relation in (b) is a relation starting and ending in one vertex $z$. By the main theorem [3] there exists a simple $\Lambda$-module $M$ with $\operatorname{Ext}_{\Lambda}^{2}(M, M) \neq(0)$. Since there are no loops in the ordinary quiver of $\Lambda$, the quiver $Q(P)$ can only consist of one cycle of length at least 2 . The claim now follows immediately from the description of the two-cornered algebras.

The three-cornered algebras have the following description. Let $P$ be a Brauerquiver with a distinguished $\beta$-loop, that is, a looped Brauer-quiver. Denote by $P^{+}$ the quiver obtained from $P$ by splitting the distinguished vertex $x$ of $P$ into an $\alpha$-sink $x^{-}$and an $\alpha$-source $x^{+}$, the arrow $\alpha: y \rightarrow x(\alpha: x \rightarrow z)$ of $P$ is replaced by an arrow $\alpha: y \rightarrow x^{-}\left(\alpha: x^{+} \rightarrow z\right)$ if $y \neq x \neq z$, if $P$ has only one vertex, the $\alpha$-loop $\alpha: x \rightarrow x$ is replaced by an arrow $\alpha: x^{+} \rightarrow x^{-}$and finally in all cases the distinguished loop $\beta: x \rightarrow x$ is replaced by an arrow $\gamma: x^{-} \rightarrow x^{+}$.

Let $P_{1}, P_{2}$ and $P_{3}$ be three looped Brauer-quivers with distinguished vertices $x_{1}, x_{2}$ and $x_{3}$. Denote by $Q\left(P_{1} P_{2} P_{3}\right)$ the quiver obtained from $P_{1}, P_{2}$ and $P_{3}$ by 
identifying the vertex $x_{2}^{+}$with $x_{3}^{-}$, the vertex $x_{3}^{+}$with $x_{1}^{-}$, the vertex $x_{1}^{+}$with $x_{2}^{-}$. Let $I\left(P_{1} P_{2} P_{3}\right)$ be the ideal in $K Q\left(P_{1} P_{2} P_{3}\right)$ generated by the elements

(a) $\alpha_{i} \beta_{i}, \beta_{i} \alpha_{i}, \alpha_{2} \alpha_{3}, \alpha_{1} \alpha_{2}, \alpha_{3} \alpha_{1}, \gamma_{3} \gamma_{2}-\alpha_{1}^{a_{x_{1}}}, \gamma_{1} \gamma_{3}-\alpha_{2}^{a_{x_{2}}}, \gamma_{2} \gamma_{1}-\alpha_{3}^{a_{x_{3}}}$, for $i=1,2,3$

(b) $\alpha^{a_{z}}-\beta^{b_{z}}$ for each $i$ and each vertex $z$ of $P_{i}$ lying outside the $\alpha$-cycle $A_{i}$ of $P_{i}$ which contains $x_{i}$.

(c) $\alpha_{i}^{a^{-}} \gamma_{i} \alpha_{i}^{a^{+}}-\beta^{b_{z}}$ for each $i$ if $z \in A_{i} \backslash\left\{x_{i}\right\}$, where $a^{-}\left(a^{+}\right)$is the length of the $\alpha$-path of $P_{i}^{+}$from $z$ to $x_{i}^{-}$(from $x_{i}^{+}$to $z$ )

If the total number of vertices in $P_{1}, P_{2}$ and $P_{3}$ is at least 5 , then the path algebra $\Lambda=K Q\left(P_{1} P_{2} P_{3}\right) / I\left(P_{1} P_{2} P_{3}\right)$ is a three-cornered algebra and all three-cornered algebras are given in this way.

Let $\Lambda$ be a three-cornered algebra. If the quiver $P_{i}$ has an $\alpha$-cycle meeting a $\beta$-cycle both of length at least 2 in a vertex $z$ of $P_{i}$ lying outside the $\alpha$-cycle $A_{i}$ of $P_{i}$ containing $x_{i}$, then the relation in (b) is a relation starting and ending in the vertex $z$.

If there is a $\beta$-cycle of length at least 2 meeting the $\alpha$-cycle $A_{i}$ in a vertex $z$ lying on $A_{i}$ and different from $x_{i}$ in $P_{i}$, then the relation in (c) is a relation starting and ending in the vertex $z$.

Again by using [3] there exists a simple $\Lambda$-module $M$ with $\operatorname{Ext}_{\Lambda}^{2}(M, M) \neq(0)$ in both cases described above. Hence each $P_{i}$ has at most one cycle of length at least 2. The claim now follows immediately from this and the description of the three-cornered algebras.

(2) Using Roggon's complete list [11] of all basic representation-finite symmetric $K$-algebras of $\Lambda$ of Dynkin type $E_{6}$ it suffices here to exhibit for each such algebra $\Lambda$ not listed in the assertion at least one indecomposable projective $\Lambda$-module $P(i)$, $i \in\{1,2, \ldots, 6\}$ whose heart $H(i)$ is decomposable. This is done in the following table.

$$
\begin{aligned}
& H(1): 2,5,6,7,9,11,15 \\
& H(2): 1,8 \\
& H(4): 4,16 \\
& H(5): 3,12
\end{aligned}
$$

(3) Using Roggon's complete list [11] of all basic representation-finite symmetric $K$-algebras of $\Lambda$ of Dynkin type $E_{7}$ it suffices here to exhibit for each such algebra $\Lambda$ not listed in the assertion at least one indecomposable projective $\Lambda$-module $P(i)$, $i \in\{1,2, \ldots, 7\}$ whose heart $H(i)$ is decomposable. This is done in the following table.

$$
\begin{aligned}
H(1): & 7,8,10 \mathrm{~s}, 11,12,13,24 \mathrm{~s}, 26 \mathrm{~s}, 27 \mathrm{~s}, 30,37 \mathrm{~s}, 38,40,43,46,51,55 \mathrm{~s}, \\
& 60,65,66,71,74 \mathrm{~s} \\
H(2): & 3,20,23 \mathrm{~s}, 31,35,48 \\
H(3): & 5,18,21,28,33,45 \mathrm{~s}, 50,54,62 \\
H(4): & 9 \mathrm{~s}, 15,22,41,56 \\
H(5): & 1 \mathrm{~s}, 4 \mathrm{~s}, 6,16 \mathrm{~s}, 25,44,47 \mathrm{~s}, 52,69,76,80 \mathrm{~s} \\
H(6): & 2 \mathrm{~s}, 14,17 \mathrm{~s}, 19 \mathrm{~s}, 29,32,34 \mathrm{~s}, 42,64 \mathrm{~s}
\end{aligned}
$$

(4) Using Roggon's complete list [11] of all basic representation-finite symmetric $K$-algebras of $\Lambda$ of Dynkin type $E_{8}$ it suffices here to exhibit for each such algebra $\Lambda$ not listed in the assertion at least one indecomposable projective $\Lambda$-module $P(i)$, $i \in\{1,2, \ldots, 8\}$ whose heart $H(i)$ is decomposable. This is done in the following table.

$H(1): 001,002,003,004,005,006 \mathrm{~s}, 007,008,009,010,011 \mathrm{~s}, 012,014$, 015, 016, 017, 018, 019, 020, 021, 022, 023, 024, 025, 027s, 028, 029, 030, 031s, 032, 033s, 034, 035, 036, 037, 038s, 039, 040, 042, 
043, 044, 046s, 049, 050, 051, 053, 054, 055, 058, 059, 061, 063, 064s, 065s, 067s, 068s, 069, 070, 071, 073, 077s, 081, 083, 084, 086, 088, 095, 096, 099, 102s, 103, 105, 106, 108, 109, 113s, 114, $117,120,126,129 \mathrm{~s}, 132,133,135,136,139,144,145,151,154 \mathrm{~s}$, $155,157 \mathrm{~s}, 160,161,162,163,168,169,171,172 \mathrm{~s}, 174,179,186 \mathrm{~s}$, $187,188,192,198 \mathrm{~s}, 203 \mathrm{~s}, 213 \mathrm{~s}, 214 \mathrm{~s}, 215,218 \mathrm{~s}, 223,224,231$, $232,233,235,240,252,253,255 \mathrm{~s}, 262,266,267,270,273 \mathrm{~s}, 284$, 290, 292, 299, 302s, 304, 308s, 314, 317, 322

$H(2): 052,056,057,074,089,090,101,104,112,147,183,191263,269$

$H(3)$ : 013s, 026s, 041, 048, 060s, 072, 079s, 082, 093, 094, 116, 125, 128, $130,134 \mathrm{~s}, 137,138 \mathrm{~s}, 140,165,189,236,247,257$

$H(4): 045,047,066,080,097,098 \mathrm{~s}, 110 \mathrm{~s}, 111,115,118,119,124,131$, 141, 148s, 170, 173, 175, 202, 206s, 211, 220, 237, 246s, 280, 283, 311

$H(5): 062 \mathrm{~s}, 076,078,085 \mathrm{~s}, 100,121,123,146,156,177,181,182,196 \mathrm{~s}$, $207,219,222,225,229 \mathrm{~s}, 238,242,271,276,300,310 \mathrm{~s}$

$H(6)$ : 087, 092, 107, 122, 127, 152s, 180, 197, 201, 204, 217, 226, 243, $256,274,291,326 \mathrm{~s}$

$H(7): 075,091,166,178,184,195,199 \mathrm{~s}, 216,239 \mathrm{~s}, 251,281,294 \mathrm{~s}, 297$, 313

Acknowledgements. Both authors kindly acknowledge financial support from the European Programme Human Capital and Mobility Grant CHRX-CT94-0601. Furthermore, the first author would like to thank the Department of Mathematics of Trondheim University for the great hospitality during his stay in April 1995. The second author would like to thank the Institute for Experimental Mathematics of Essen University for very inspiring working conditions during his stay in March 1995.

\section{REFERENCES}

[1] M. Auslander, S. Ding, Ø. Solberg, Liftings and weak liftings of modules, J. Algebra, 156, (1993) 273-317.

[2] M. Auslander, I. Reiten, S.O. Smalø, Representation theory of Artin algebras, Cambridge Univ. Press, Cambridge, 1995.

[3] K. Bongartz, Algebras and quadratic froms, J. London Math. Soc. (2), 28 (1983) 461-469.

[4] O. Bretscher, C. Läser, C. Riedtmann, Selfinjective and simply connected algebras, Manuscripta Math. 36 (1981) 253-307.

[5] W. Feit, The representation theory of finite groups, North-Holland, Amsterdam, 1982.

[6] J.A. Green, A lifting theorem for modular representations, Proc. Roy. Soc. London, Ser. A. (1959) 135-142.

[7] D. Hughes, J. Waschbüsch, Trivial extensions of tilted algebras, Proc. London Math. Soc. 46 (1983) 347-364.

[8] G.J. Janusz, Indecomposable modules for finite groups, Annals of Math. 89 (1969), 209-241.

[9] H. Kupisch, Symmetrische Algebren mit endlich vielen unzerlegbaren Darstellungen I, J. reine u. angew. Math., 219 (1965), 1-25.

[10] H. Kupisch, Quasi-Frobenius algebras of finite representation type, Springer Lecture Notes in Math. 488 (1975), 184-200.

[11] B. Roggon, Selfinjective and iterated tilted algebras of type $E_{6}, E_{7}, E_{8}$, Preprint, Math. Fak. Univ. Bielefeld (1994).

[12] A. Skowronski, J. Waschbüsch, Representation-finite biserial algebras, J. reine u. angew. Math. 345 (1983), 172-181.

[13] J. Waschbüsch, Symmetrische Algebren vom endlichen Modultyp, J. reine u. angew. Math., 341 (1981), 78-98.

Institut Für eXPerimentelle Mathematik, Universität GH Essen, Ellernstr. 29, 45326 Essen, Germany 
THE REPRESENTATION FINITE SYMMETRIC. .

Institutt FOR MATEMATIKK OG STAtistikk, Universitetet i Trondheim, AVH, N-7055 Dragvoll, Norway 